\title{
Implementing a mixed methods Human Factors approach to design and rehearse management of unplanned extubation in critically ill adult patients
}

Alexandra Rosewall Lang ( $\nabla$ alexandra.lang@nottingham.ac.uk)

University of Nottingham Faculty of Engineering https://orcid.org/0000-0002-7332-9443

Bryn Baxendale

Nottingham University Hospitals NHS Trust

Sarah Atkinson

University of Nottingham Faculty of Engineering

Jeena Velzen

Memorial Sloan Kettering Cancer Center

Thomas Blanks

Nottingham University Hospitals NHS Trust

Umair Ansari

University Hospitals Coventry and Warwickshire NHS Trust

Mark Kane

Nottingham University Hospitals NHS Trust

Giulia Miles

Nottingham University Hospitals NHS Trust

Thearina DeBeer

Nottingham University Hospitals NHS Trust

Steven Gill

Nottingham University Hospitals NHS Trust

\section{Research}

Keywords: Critical care, Human Factors, Patient Safety, Airway Management, Unplanned Extubation, Qualitative methods, Simulation

Posted Date: August 5th, 2020

DOI: https://doi.org/10.21203/rs.3.rs-52229/v1

License: (a) (i) This work is licensed under a Creative Commons Attribution 4.0 International License. Read Full License 


\section{Abstract}

Background: Unplanned extubation (UE), the unintentional dislodgement of a patient's artificial airway; indwelling endotracheal tube or tracheostomy, occurs infrequently in the critical care setting but is a high risk incident and requires appropriately skilled staff and clear management plans to manage and ensure a positive outcome for the patient. Within the current guidance on management of UE, there is superficial consideration of the systems view and limited practical advice about the integration of systems thinking into management of these situations.

Method: This paper reports the findings of a multidisciplinary Human Factors (HF) study to understand 'work as done' in being prepared and responding to UE in practice. Multiple methods were triangulated to take a systems perspective on these situations. The methods included development of a hierarchical task analysis (HTA), 21 expert interviews, 50 equipment familiarity audit surveys and three in situ simulation exercises.

Results: The study identified key areas of focus for redesign of environment and accessibility of equipment and organisation of resources. It was established that difficulties in identifying individual competency and team skill mix appropriate to the scenario requirements undermined people's nontechnical skills and that decision making could be negatively impacted as a result.

Conclusion: The HF approach has provided the critical care team with an improved understanding of the work involved in being prepared or responding to UE and a visual task breakdown which can be used for identifying and testing new ways of working, different environments, technological interventions and using simulation to rehearse for performance. The findings will inform future changes to practice, design of work and the development of a mastery learning programme to support individual and team capabilities in responding to UE so that staff are able to consider this infrequent scenario in context of the wider system.

\section{Introduction}

This paper describes a study undertaken at a large acute teaching hospital in the United Kingdom (UK), where clinical practices associated with unplanned extubation (UE) on an adult critical care were in need of review and improvement. In response to this statement of need a multidisciplinary research team of clinical staff (consultants and registrars) human factors and simulation experts was established to investigate current practices in the adult critical care environment and the issues being experienced.

The aims of the study were to,

- Improve understanding about Work as Done (WAD) as opposed to Work as Imagined (WAI) [1] regarding management of UE in the adult critical care setting.

- Provide recommendations for system improvement and redesign to support UE management and proactive safety practices 
Over the last couple of years there has been increasing evidence and call for the integration of Human Factors (HF) and systems thinking into health and social care $[2,3,4]$ and specifically critical care environments [5,6]. It has been established that there is an important role for this discipline [7], to support and educate health service design improvement, evidenced based procurement [8] and evaluation of safety, quality and wellbeing within the system $[9,10]$.

The iterative and participatory HF approach adopted for this study produced outputs to support opportunities for redesign of work and space in adult critical care environments. In addition, these insights into the requisite technical and non-technical skills and team-based behaviours that underpin WAD, would support development of a simulation based Mastery Learning Programme (MLP), to deliver proactive risk assessment and develop simulation activities which are cognisant of context and how the clinical scenario may be experienced within the wider system. The importance of this work, its findings and application are highly relevant to the field, as the base specialty for entering critical care medicine is moving away from anaesthetics, to medical specialties, resulting in fewer critical care medical staff being airway trained.

\subsection{UEs and Critical Care}

One aspect of critical care medicine provided to patients in adult critical care is mechanical ventilation. To achieve this ventilation there is a requirement for the insertion of either an oral endotracheal or a tracheostomy tube via the anterior neck. This study collected data from cases of endotracheal intubation UE's and examples of tracheostomy UE. Within the local hospital trust in which this study took place, on average 1-2 UEs occur per week.

UE refers to the inadvertent or unintentional extubation (removal of artificial airway support) of a patient prior to intended removal. UE events can be categorised based on the causative mechanism, whether the extubation is intentionally or unintentionally actioned by patients or staff $[11,12]$. There is no clear protocol specifically for the management of UE on adult critical care, although various bodies provide guidance on extubation such as the Difficult Airway Society (DAS) $[13,14,15]$.

UE remains one of the most common complications of endotracheal intubation with studies reporting ranges of incidence between $2 \%$ [16] to $14 \%$ [17], up to $35 \%$ [18] of ventilated patients. There is conflicting evidence with regard to UE association with increased mortality $[19,20]$ and for patients that require reintubation, there is evidenced impact on; length of mechanical ventilation, increased ventilator associated pneumonia, longer critical care (CC) and general hospital stay and an increased requirement for on-going chronic care [20,21]. The literature provides a backdrop of understanding about where UEs occur and who has to deal with them. High proportions of CC airway events were reported in the UK based National Audit Project 4, detailing UE occurrences at night and how they were managed by junior CC staff [22] and an additional survey showed that $32.3 \%$ of CCs had uncertain levels of cover with airway-trained staff during out-of-hours [23].

\subsection{Human Factors for health system improvement and design}


A UE scenario creates a time and safety critical situation for often inexperienced staff within a clinical multidisciplinary team; needing to re-secure the patient airway for a positive outcome. Time-pressured environments are known to be challenging to human decision making in safety critical industries [24, 25] and this issue is pertinent to $\mathrm{CC}$ as the administration of medications and procedures is complicated by the acutely unwell nature of the patient.

Historically in healthcare there has been a tendency for serious incident investigations and subsequent recommendations and improvement strategies to focus on non-technical skills and rely on training as a means of promoting safety in healthcare. HF works to the hierarchy of control as [26], first and foremost looking to redesign system elements to 'design out' potential errors, help users do the right thing easily and develop system resilience rather than rely disproportionately on 'human resilience' through training interventions $[10,27]$.

\section{Methods}

A protocol was designed to collect data for triangulation, to get a full picture of WAD vs WAI. An analysis of human, situational, equipment and environmental factors inherent in management of UE was explored via four data collection activities. Multiple data sources informed the development of a Hierarchical Task Analysis (HTA) [28], which provided a resource for use in semi-structured staff interviews capturing experience of UE events. In-situ simulation exercises were generated from the interview data to observe how staff responded to specific UE scenarios in three different critical care environments. Finally, a survey of airway equipment familiarity was implemented to understand staff awareness of and familiarity with equipment and location of equipment used in these infrequent situations. The data collection period spanned 4 months and was iterative in nature. To ensure there were no conflicts of interest with clinical practice, the HF team led the design of the protocol and the elicitation of experiential data from the critical care staff. The in situ simulations were led by the simulation experts.

Following consultation with the local Research and Innovation department and utilising the Medical Research Council decision tool [29] the study was considered eligible for classification as a Service Improvement study and so did not require ethical review.

\subsection{Hierarchical Task Analysis}

The HTA was developed by HF experts representing the sequence of operations performed in a UE event. The task analysis was generated using local Standard Operating Procedures (SOPs) and available national guidance $[13,15]$ to present a model of WAI. SOP's and guidance documents provide generic guidance for managing these scenarios, the context in which they occur, staff available and other parameters mean that there will be variation in the way that they are experienced and managed. The HTA was then supplemented by interviews with two subject matter experts in order to produce a visualisation more closely representing WAD.

Figure 1. Hierarchical Task Analysis of Unplanned Extubations 


\subsection{Semi-structured interviews}

Semi structured interview schedules were then developed using the HTA (figure 1) and Onion Model of Interactions [30] to investigate how UE's are experienced by frontline staff, moving to a closer understanding of WAD. A total of 21 CC qualified staff including nurses $(n=11)$ and doctors $(n=10)$ were interviewed, (Table 1), the duration of the interview ranged from 18 to 68 minutes.

Table 1. Interview Participants

\begin{tabular}{|l|l|l|l|}
\hline \multirow{2}{*}{ Nurses } & Junior <3 years in CC (NJ) & Senior 3+ years in CC (NS) & Total \\
\cline { 2 - 4 } & 5 & 6 & 11 \\
\hline \multirow{2}{*}{ Doctors } & Non-Airway trained (DN) & Airway Trained (DA) & Total \\
\cline { 2 - 4 } & 5 & 5 & 10 \\
\hline
\end{tabular}

Participants from CC multidisciplinary teams (MDTs) were presented with a copy of the HTA at the interview. The interviews were conducted by a HF researcher, which enabled CC staff to be candid in their accounts about UE WAD, disclosing information which they might not have shared had they been interviewed by a member of staff from the same organisation. Each interview was recorded digitally, with consent.

\subsection{Survey of staff familiarity with airway equipment}

50 CC staff were surveyed (nurses $n=38$, medical $n=12$ ), with a median critical care experience of 14 months. Participants were asked to locate and identify UE equipment from an airway trolley and demonstrate how to prepare and safety check the equipment. A binary metric of 'correct' or 'incorrect' identification was used to assess individual's familiarity with and ability to locate equipment on the airway trolley.

\subsection{Simulations}

Simulation exercises were conducted in three CC environments, using an adult high-fidelity simulation manikin and mobile network video recording system including base camera units, Go-Pro ${ }^{\mathrm{TM}}$ and wearable recording glasses. Each scenario was designed by a simulation faculty with subject matter expertise based on reported UE incidents and airway management on CC. Multidisciplinary teams on CC wards were informed in the morning briefing that simulation activities were going to occur during their shift but were not informed about what scenario they would be presented with or when. Box 1 provides an example in-situ simulation scenario used in the study. The video glasses were provided to the UE clinical lead at the point of the simulation occurring. 


\subsection{Data Analysis}

The interview data was analysed using inductive emergent thematic analysis [31] by a HF expert using NVivo ${ }^{\mathrm{TM}}$ software.

Analysis of the three simulation video recordings involved a working group of three HF experts and three clinical staff experienced in critical care airway management practices. This analysis used predetermined categories (shown in Table 2)

Table 2. Analysis categories for observed behavior/ actions during simulation

\begin{tabular}{|l|l|}
\hline \multicolumn{1}{|c|}{ Clinical categories } & \multicolumn{1}{c|}{ HF categories } \\
\hline Recognition and escalation of incident & Tasks \\
\hline Responding team & People \\
\hline Team actions & Information \\
\hline Environment and Resources & Equipment and Environment \\
\hline
\end{tabular}

Videos were reviewed concurrently and recordings were paused for clarification on process, tasks and job roles. Videos were observed and discussed in relation to expected practice, the interview findings and areas for improvement.

Survey scores relating to familiarity of airway equipment were analysed with regard to job role (nursing or medical participant).

\section{Results}

The following section describes the results from each method and the cumulative learning from the data are reported in the discussion section. The triangulation of the data sets enabled:

- $\quad$ Understanding of the interactions occurring throughout the scenario

- Identification of issues associated with design of tasks, equipment and environment

- $\quad$ Evaluation of individual and team working practices and decision making

- $\quad$ Representation of the system of work as WAD

All 21 participants were asked to share their experiences of dealing hands-on or in supporting the management of an UE. The details of these scenarios are shown in Table 3.

Table 3: Participants reflections on UEs 


\begin{tabular}{|l|l|}
\hline Cause of UE Incident & Number of Participants \\
\hline Self-extubation & 10 \\
\hline Moving or turning the patient & 8 \\
\hline Unknown & 4 \\
\hline Not secured & 3 \\
\hline Type of Intubation & Number of Participants \\
\hline Tracheostomy & 13 \\
\hline Oral intubation & 9 \\
\hline Time of Occurrence & Number of Participants \\
\hline Night time & 9 \\
\hline Day time & 5 \\
\hline
\end{tabular}

\subsection{Review of HTA}

Participant responses confirmed that the HTA was an appropriate representation of how UEs are mostly experienced.

One task not identified in the original UE HTA was the application of cricoid pressure, which 8 participants mentioned. However this step, of applying pressure to the cricoid cartilage was noted as being controversial in its effectiveness, "It's an area of slight controversy... it's something that is done not very much at all in some countries... there's some controversy in exactly how effective it is." (DA-15). The cricoid pressure is not removed until the doctor has checked the final placement of the ETT.

Additional tasks suggested for inclusion in the HTA and could be included in future versions, include but are not limited to; settle patient, position patient, turn off alarms, bronchoscopy, check cuff pressure.

\subsection{Organisation during UE}

Interviewees spoke of ways they might recognise a UE incident in a patient through visually identifying tube displacement, hearing auditory signs (i.e. gurgling of secretions or air cuff leak) or seeing or hearing alarms (i.e. apnoea/desaturation alarm or ventilator alarm). These cues are the impetus for the first priority of alerting other colleagues to the situation. The findings suggest that the design of the environment can impact how help is obtained and how quickly it attends. In an open ward environment participants (13) made reference to calling out for help. One airway doctor said, "... they would just shout across the floor that they need help, for someone to come and see what's going on" (DA-8). In units with isolated side rooms, other strategies occur to attract attention, such as banging on the door or pressing emergency call buttons which are audible outside of the side room. Challenges were described in regard 
to using and the effectiveness of call buttons "it may be difficult to get to the buzzer if patient is agitated and it's not ideal, because... I practiced 3 times and barely anyone came."(DA-21). Additional points were raised by participants regarding the environment of care, specifically how dimmer lighting at night can impact emergency procedures and how noisy units may make it difficult to discern or distinguish between alarms. These environmental challenges were evidenced first-hand within the in-situ simulations, with staff struggling to gain the attention of colleagues due to the ward design and poor design of call buzzers.

A majority of interviewees (15) made specific reference to pre-emptive "gathering" of the airway emergency trolley for an UE. This trolley contains the necessary intubation and emergency airway supplies. Recently, an intubation bag was devised which contains the basic equipment necessary for intubation and rests on the arrest trolley. "So here the nurses are quite hands on and they were proactive, so one of them immediately went to get the arrest trolley with all of the equipment." (DA-20). However, this response may be dependent on experience of the nurses, "It depends on the experience of the nurse cos some nurses would know immediately what to get, how to get it, what to do, but some don't really know" (DA-9). Similarly, the collection of appropriate drugs for reintubation was considered variable based on the location of drugs in a secure store only accessible by specific personnel. During the simulations, the trolley was collected quickly for each scenario, however it was apparent that in some cases, the use of the equipment from the trolleys was challenging due to lack of space around the bedside. Staff developed coping strategies to account for the environmental limitations e.g. putting equipment on the bed or patient. This occurred in parallel with other task requirements of a UE, specifically gaining access to the head end of the bed and lowering the patient to a horizontal position for airway management.

Participants made clear that the default course of action for an UE is reintubation, often quickly discernible for both nurses and doctors. An airway doctor explained "if it wasn't someone who was planned to extubate I think I would just carry on and intubate the patient. You can always extubate again as long as you have a safe airway." (DA-9). While the decision to reintubate is an airway doctor led decision, some participants stated that they would seek more senior advice in either consultation or assistance with the CC consultant or the on-call anaesthetist. Interview responses demonstrated that nurses are also able to participate in the decision-making process "if... the doctor wanted to reintubate, I would have been quite firm and said, 'Look, you obviously can. I can't stop you, but I really do recommend that we just let her settle and let her see how she does.' And to be fair, on critical care the doctors are really good. They really do listen to us and respect what we say, which is good" (NJ-6).

The readiness and checking of intubation equipment at the patient bedside is an important step before clinical intervention. "They would... get the things that were needed and check them, open the ET tube, check the balloon, open the lubricant, check the laryngoscope, check you've got a bougie, check that the suction's to hand for the doctor and it's where you need it"(NS-1). This was supported by participants' accounts of equipment safety checks which are carried out twice a day. 
Interview responses suggested that there is no standard lay-out procedure for equipment to support efficient work and decision making. Only one junior nurse made reference to the "intubation mat" stored on the arrest trolley which helps to ensure all supplies are collected and laid out in order, a potentially useful resource that was not used in the simulations either. "You have your mat... and you put all the equipment on the cut out of the mat... it sounds really silly and really simple, but it's really, really good... It's just like one of those jigsaw puzzles that you've got the gap in, so you know you've got everything, because there's nothing left on the mat. The doctor... knows exactly where everything was so they can practically do it blindfolded." ( $\mathrm{NJ}-6)$.

Preventing delay was considered a significant factor in a smooth UE management process, with participants considering the preparedness of equipment and drugs and availability of appropriate personnel to be key indicators of readiness to deal with an emergency situation.

\subsection{Experience and skills mix}

There was a discrepancy between assumed competencies based on role in the organisation, particularly airway management. Interviews of doctors revealed an assumption that nursing staff will know from experience which people are airway trained "I believe they know who are airway trained or not here, I think there are few senior clinical folks who are not, who are respiratory trained so they know who can and cannot manage the airway" (DA-8). It was clear during the simulations that teams were not aware of each other's skills set and no explicit role allocation was observed during the simulations. There was an informal process of self-allocation to tasks based on environmental challenges in the design of the area. This lack of knowledge about colleague's experience related specifically to identification of an airway trained doctor in the $\mathrm{CC}$, with assumptions of competency being based on seniority.

The interviews and in-situ simulations highlighted that there was no formal process to identify those with airway competency, despite this being an essential step. One nurse described her practice for identifying an appropriate person in this role, "Well you should ensure that... at the beginning of the shift, to know who is the doctor is airway trained and if you know that you don't have that you can dial double 2, double 2 and then call for an airway trained doctor." (NJ-13). This issue was further reported regarding the general skills mix within the teams "I think it's really hard, because you don't always know the nurse you're working with and what their skills are and what their abilities are and you can over-estimate or underestimate them so it's quite, quite difficult" $(\mathrm{NJ}-6)$. A reliance on informal and serendipitous learning to establish peer experience and competence was widespread throughout the sample population.

Participants identified the immediate need to oxygenate the patient. This task highlighted how variation in staff experience may impact action in the early stage of an UE. Airway doctors suggested that some nurses may have varying capabilities in providing airway and breathing support using a face mask and self-inflating bag otherwise known as 'bagging', "It depends on the competencies of the nurses who are looking after the patients, obviously some of the nurses are happy to try and bag and mask themselves" (DA-8) and this perspective was replicated for non-airway trained doctors "A lot of their focus is on calling for help. And they do try to bag and mask the patient, but most people's bag and mask skills are very 
poor" (DA-21). This latter point was experienced in one of the simulations where a less experienced junior doctor (non-airway trained) was present for the UE simulation and had to be prompted and coached through several actions to involve them in the process.

Participants discussed the number of people at the bedside supporting an UE, most suggested having fewer people immediately attending, as too many people can cause confusion. "Sometimes it can get a bit crowded when everyone wants to help and there have been situations... when you just look around and you think, there are too many people. And if there are too many people, you kind of lose track of who's doing what. So normally I try and keep the number of people around to how many we need, otherwise it just gets chaotic." (DA-20). This was perceived by both doctors and nurses "I know they're only there just in case, but I think sometimes when there's too many people there you don't know who's doing what, so I think there needs to be the right amount of people that have their own jobs and not people lingering." (NJ19).

The interviews showed a tendency for a medical doctor to be the individual allocating tasks and leading the situation, with nursing staff asking for task direction. However, in the simulations, senior nursing staff also played key roles in allocating tasks and coordinating activities. There was a strong appreciation for the dynamic and flexible nature of the leadership role although there was little consensus upon any approach to leadership handover.

\subsection{Communication and information transfer}

Immediate communication with the lead clinician would be provided by the attending nurse. Doctors were most interested in knowing what had happened and a brief patient background, to help determine a course of action. Apart from relevant patient history, such as relevant surgical information, participants (8) described the need to know the previous intubation grade, provided in the medical notes. "I normally like to know whether they were previously difficult or not, or easy intubation, and what grade of intubation, whoever previously did it noted, because that will... it will alter my approach."(DA-20).

Prior knowledge of a patient was considered a factor in the time required in determining an appropriate airway management plan and therefore handovers and prior experience with a patient were deemed to be important in UE management. This was referenced in regard to communication within the team and also external stakeholders who might be involved in the event of a UE "... sometimes the anaesthetist that we would call in an emergency if the consultant wasn't there, they started to come round our unit and they'd been made aware of high risk patients and he would have been highlighted to the anaesthetist that evening before, before the consultant left."(DN-7).

When recollecting episodes of well managed UE events, there was a consistent theme regarding clear task delegation and allocation to individuals involved. This was at odds with the observed behaviour in the simulation activities where there was little use of positive verbal affirmation of information receipt or in the use of language to directly communicate a task with a specific individual. 


\section{Discussion}

The combination of data from the methods utilised provides signposting for system improvements and data around WAD to support the development of a Mastery Learning Programme.

\subsection{People}

The initial gathering of critical information over a short period of time once a UE had been identified was reported in the interviews and evidenced in the simulations to occur efficiently and comprehensively, as was the collection of the airway trolley.

There is a requirement for a team with mixed skills and experience to respond to a UE. However, it was evident that MDTs find it difficult to determine the skill set available in these time-critical situations and as such there is no guidance or structure for the roles that personnel can take on during a UE. One exception to this is the automatic need for a team lead in this time critical scenario, a role most often taken on by the most senior medical personnel present. The identification of roles occurs as a result of implied competence related to seniority as an informal and tacit process rather than explicit identification. It is considered that with current CC's being increasing manned by medically trained personnel rather than airway trained personnel, that there is the creation of a new role 'airway assistant' who can support senior airway trained clinicians but also coordinate and manage prior to arrival of more experienced personnel. To standardise and facilitate development of this role, it is advised that it is developed and linked with core competencies as prescribed by mandating professional bodies e.g. British Association of Critical Care Nurses (BACCN) and the Difficult Airways Society (DAS).

\subsection{Equipment and Environment}

The current system and environment does not always support efficient 'raising of the alarm' and obtaining help from colleagues in an UE event. This task is hampered by variability in the ways (calling for help, banging on doors) and mechanisms (buzzers, pull cords, etc) that staff use to summon help. The design of the environment can be an enabler for quicker, easier alerting for UEs and other emergency situations.

The survey and simulation results highlighted that identification of critical equipment in the UE could be improved and that staff are not as familiar with the airway trolleys as they self report. This finding supports the work of Schnittker et al [32] who identified that the design of airway trolleys could be a barrier to action and decision making. The existing airway mat provides a tool to support staff in faster identification and selection of equipment for use however staff were not familiar with this resource. There is a recommendation for this item to be designed into the trolley in a way that promotes its use in the event of an UE or to procure trolleys for which this is already the case.

This study has identified that the environment in which UE's take place is often not suitable for those emergency scenarios and could potentially be detrimental to team organisation, response time, standardising the approach to UE's and use of resource (person, equipment and consumables). 


\subsection{Systems and Policy}

The lack of a local, regional or national protocol for the clinical management of this event leads to widespread variation in the frontline management and reporting of UE. Without a clear plan for how to manage these situations, and therefore identifying the skills and personnel needed, there is little consensus as to what is good or bad practice other than an outcome-driven goal of management and reporting. It is easy to conceive that a recognised UE, which is managed promptly and safely by the healthcare team, may not be regarded as 'reportable' because the positive outcome of the event detracts from the process of the UE. This is not a new phenomenon in healthcare and therefore using process measures to draw inferences about the quality of care further upstream is commonplace [33]. In addition to the normal utilisation of process measures, this study advocates the use of a systems approach to consider the wider contextual issues that impact UE practice. The understanding of system influencers on processes and people's behaviour can provide tangible information signposting towards specific areas for improvement through design.

\subsection{Future work for a WAD derived Mastery Learning Programme (MLP)}

MLP (related to Competency based learning) is defined as "an especially stringent form of competencybased education where learners acquire essential knowledge and skill measured rigorously against fixed achievement standards without regard to the time needed to reach the outcome" [34].

This method of learning is evidenced as an effective educational intervention when appropriately initiated in medical fields [35, 36]. However, MLP is often derived from expert consensus and as such can be far removed from real world context in which is it applied. The findings from this study provide a 'real world' comprehensive perspective about the interactions of staff in the event of a UE, with acknowledgment of service, system and environmental variations and as such provide a robust data set from which to develop an effective UE focused MLP that is based in WAD not WAI. This representation of WAD can then be tailored within the MLP, using routine extubations to consider 'what if scenarios', varying skills mix and patient acuity, working towards a programme of proactive risk management and patient safety which is embedded in a systems thinking approach.

The Royal College of Anaesthetists recommend the use of MLP with simulation [37], it is considered that this would be further enhanced through the additional integration of HF methods to ensure an MLP which is inclusive of WAD in context and system considerations, cumulatively providing a more powerful and effective training intervention than is currently on offer.

\subsection{Limitations}

This study has only currently involved CCs from one teaching hospital in the UK. To understand the experiences and ways of improving UE practice, a wider sample needs to be involved from a range of services/ care facilities. Recruitment of larger numbers of consultants and involvement of anaesthetists 
is recommended to support a more comprehensive view on UE practice and any subsequent intervention design.

A limitation of this research was the retrospective nature of the interview data, as participants were required, if applicable, to recall past experiences of managing UEs in situ. Due to the variation of experience for participants and the irregularity (for some) of managing a UE, interviews relied on best recollection of events and factors involved. A visual HTA prompt was provided to enhance recall of various aspects of participant UE experiences.

Despite efforts, the team was unable to retain a nurse representative for the duration of the study. This means that in addition to the researcher bias which may be present, there is likely a medical bias which is not inclusive of a nursing viewpoint or indeed a service user perspective.

Technical limitations such as access, availability of power supply dictated some aspects of the simulation e.g. where the video base units were placed to record the UE simulations. Additionally, the limited number of scenarios only occurred during day shifts and so there will be a bias in the data towards CC wards that are resourced for day and not representative of a reduced nightshift workforce. Despite the evidence for positive value derived from inter-professional in-situ simulations [38] there are some limitations to what was experienced in this study which are common across simulation activities, for example the bias associated with awareness of being monitored and the inference of 'leadership' designated to medical personnel due to the provision of the wearable glasses by the simulation team.

\section{Reflections On Research Process}

The broad range of expertise applied to the study was a strength of the approach, and as such it is recommended that this model be used in future investigations into the safety and performance within CC environments.

The recommendation of integrating input from a HF 'Suitably Qualified and Experienced Person' (SQEP) [10] with clinical and simulation experts provides a systems design lens to the problem and rigorous methods. Additionally, this non-clinical independent perspective adds value to the team by enabling the team to go back to basics and remove any assumptions about WAD. This approach is supported in the literature [39], carefully implemented interventions, such as checklists, new processes and equipment along with human factors engineering has the potential to improve teamwork, communication, safety and efficiency of care.

\section{Conclusion}

This research enquiry has been underpinned by established HF principles and was designed to investigate the system of work around UE specifically and airway rescue more generally. 
The HTA provides a resource from which to understand the overall procedure, sub tasks and decision making points in the management of airways rescue and provided a mechanism to facilitate interviewees to consider the enquiries in a structured manner, without assumptions.

The breadth of information from this study provides a deeper understanding of the CC nurse and doctor experience in UE response and management, and uncovers the variances to practice and key areas which can be carried over into best practice recommendations. It has provided signposting to design changes for the improvement of UE experience by multiple stakeholders. The data elicited provides valuable insight into the non-technical and technical skills and contextual requirements of UE practice to inform the development of a MLP for the mental and practical rehearsal of UE, in addition to review of planned extubation practices. Importantly this training is being developed to be inclusive of a HF and systems lens on a potential UE scenario.

This study has triangulated experience from multiple disciplines and data from multiple methods to develop a strong, evidenced based approach to understanding WAD of UEs in CC. This findings of which are being used to inform local hospital system improvements and will be further utilised in the production of an MLP that is based on WAD. This approach aligns with but also goes further than the current DAS guidance [15]. The DAS resource advocates HF inclusion but does not prescribe incorporating design of the work system e.g. the need for an airway assistant role, design and organisation of workspace and equipment, as part of continuous assessment and improvement. As such this paper looks to promote an understanding of HF application which goes beyond a tokenistic application, aiming for integrated approaches which optimise the value of HF [7] to quality and safety improvement strategies in critical care.

The study provides an example of a pragmatic approach to understanding WAD of UEs in CC. It has demonstrated the benefit of interdisciplinary specialties working in collaboration to work towards system led improvements for UE scenarios and airway management generally.

\section{List Of Abbreviations}

UE - Unplanned Extubation/ WAD - Work as Done/ WAI - Work as Imagined/ CC - Critical Care/ HF Human Factors/ MLP - Mastery Learning Programme / HTA - Hierarchical Task Analysis/ SOP Standard Operating Procedure / MDT - Multidisciplinary Teams / ETT - Endotracheal Tube / DAS Difficult Airways Society / SQEP - Suitably Qualified Experienced Person

\section{Declarations}

Ethics approval and consent to participate: Ethical approval for this study was waived under an existing agreement between the Hospital Trust and academic institution involved, whereby proposed research projects identified as contributors to 'Service Improvement' which do not have any patient involvement can proceed without ethics approval. 
Consent for Publication: Not Applicable

Availability of data and materials: The dataset(s) supporting the conclusions of this article are available in from The University of Nottingham data repository.

Competing Interests: The authors declare that they have no competing interests.

Funding: The authors acknowledge the funding of this work by Health Education England working in the East Midlands, under Project ID: HFNOT004. The funder had no involvement in study design, data collection, analysis, interpretation, writing or decisions involving publication.

Authors' contributions: Conceptualisation and Funding acquisition: BB, SA, AL \& GM Methodology: AL, SA, BB. Project administration: GM. Resources: SG \& MK. Data curation: JV, TB, UA. Simulations: BB, MK, SG, TB \& UA. Formal analysis: JV, UA, AL, SA, BB, MK and SG. Supervision and Validation: SG \& TdB. Writing original: AL. Writing reviewing and editing: BB, SA, SG, TdB, TB, GM, JV, MK, UA. All authors read and approved the final manuscript.

Acknowledgments: We would like to thank the clinical participants who took part in the data collection activities for this study. We would like to thank the Research and Innovation Department at Nottingham University Hospitals Trust for their advice and guidance pertaining to ethical approvals for this study.

\section{References}

1. Hollnagel E (2017) Why is Work-As-Imagined different from Work-As-Done? Chapter 18. Eds.Wears RL \& Hollnagel E. Resilient Healthcare Vol 2. CRC Press. London.

2. Sevdalis N, Brett, S.J. (2009) Improving care by understanding the way we work: human factors and behavioural science in the context of intensive care. Crit Care 13, 139 (2009).

3. NCB - National Quality Board. (2013) Human Factors in Healthcare. A Concordat.http://www.england.nhs.uk/wp-content/uploads/2013/11/nqb-hum-fact-concord.pdf. Accessed: 06/08/2019.

4. Carayon P, Wooldridge A, Hose BZ, et al. (2018) Challenges And Opportunities For Improving Patient Safety Through Human Factors And Systems Engineering. Health Affairs. 37:11

5. Rubulotta, F., Scales, D.C. \& Halpern, S.D. Night shifts, human factors, and errors in the ICU: a causal pathway? Intensive Care Med 42, 456-457

6. Hamilton DK (2020) Ch7 Design for Critical Care. In Design for Health. Eds Sethumadhavan A \& Sasangohar F. Academic Press. Pp 129-145.

7. Chartered Institute of Ergonomics and Human Factors. (2018). Human Factors for Health \& Social Care (White Paper). Birmingham: CIEHF. Available at https://www.ergonomics.org.uk/Healthcare

8. Ginsburg G (2005) Human factors engineering: A tool for medical device evaluation in hospital procurement decision-making. Journal of Biomedical Informatics. 38:3. Pp 213-219 
9. Pickup L, Lang AR, Atkinson S \& Sharples S (2018) The dichotomy of the application of a systems approach in UK healthcare the challenges and priorities for implementation. Ergonomics. 61:1. 15-25.

10. Hignett S, Lang AR, Pickup L, et al. (2018) More holes than cheese. What prevents the delivery of effective, high quality and safe health care in England?, Ergonomics, 61:1, 5-14, DOI: 10.1080/00140139.2016.1245446

11. Chevron V, Menard JF, Richard JC, et al. (1998) Unplanned extubation: risk factors of development and predictive criteria for reintubation. Crit Care Med ;26:1049-1053.

12. Moons P, Sels K, De BW, et al. (2004) Development of a risk assessment tool for deliberate selfextubation in intensive care patients. Intensive Care Med;30:1348-1355.

13. Popat M, Mitchell V, Dravid R, (2012) Difficult Airway Society Guidelines for the management of tracheal extubation. Anaesthesia. 67(3):318-340

14. Frerk C., Mitchell V.S, McNarry A.F et al.(2015) Difficult airway society 2015 guidelines for management of unanticipated difficult intubation in adults. British Journal of Anaesthesia. 115, 827-848

15. Higgs A, McGrath BA, Goddard C et al (2018) Guidelines for the management of tracheal intubation in critically ill adults. British Journal of Anaesthesia, 120 (2): $323 e 352$

16. De Groot RI, Dekkers O, Herold I, et al. (2011) Risk factors and outcomes after unplanned extubations on the ICU: a case-control study. Critical Care,15:R19.

17. Mion L, Minnick A, Leipzig R, et al. (2007) Patient-initiated device removal in intensive care units: A national prevalence study. Crit Care Med;35(12):2714-2720.

18. Da Silva PSL, Fonseca MCM (2012) Unplanned Endotracheal Extubations in the Intensive Care Unit, Anesthesia \& Analgesia; 114:55 - p 1003-1014

19. Jaber S, Amraoui J, Lefrant JY,et al. (2006) Clinical practice and risk factors for immediate complications of endotracheal intubation in the intensive care unit: a prospective, multiple-center study. Crit Care Med;34(9):2355-61.

20. Epstein SK, Nevins ML, Chung J. (2000) Effect of unplanned extubation on outcome of mechanical ventilation. Am J Respir Crit Care Med,161(6):1912-1916.

21. Epstein SK, Ciubotaru RL, Wong JB. (1997) Effect of failed extubation on the outcome of mechanical ventilation. Chest,112:186-192.

22. Cook TM, Woodall N, Harper J, et al. (2011) Major complications of airway management in the UK: results of the Fourth National Audit Project of the Royal College of Anaesthetists and the Difficult Airway Society. Part 2: intensive care and emergency departments. Br J Anaesth;106:632-42.

23. Astin J, King EC, Bradley T, et al. (2012) Survey of airway management strategies and experience of non-consultant doctors in intensive care units in the UK. Br J Anaesth;109(5):821-825.

24. Redmill F \& Rajan J (1997) Human Factors in Safety Critical Systems. Butterworth- Heinemann, Oxford UK. 
25. Cohen I (2008) Improving Time-Critical Decision Making in Life-Threatening Situations: Observations and Insights. Decision Analysis. 5;2:100-110.

26. Sanders, M. and McCormick, E. (1998), Human Factors in Engineering and Design. $7^{\text {th }}$ Edition. McGraw-Hill New York.

27. Russ AL, Fairbanks RJ, Karsh B, et al. (2013) The science of human factors: separating fact from fiction. BMJ Quality \& Safety,22:802-808.

28. Kirwan, B. and Ainsworth, L.K. eds. (1992) A Guide to Task Analysis: The Task Analysis. Boca Raton, London, New York: Taylor \& Francis.

29. Medical Research Council, National Health Service Health Research Authority Is my study research? [online] Available at: http://www.hra-decisiontools.org.uk/research/ [Accessed June 2018].

30. Wilson, JR \& Sharples S. (2015) Method in the Understanding of Human Factors. In JR Wilson \& Sharples (Eds). Evaluation of Human Work: 4th Edition. Boca Raton, London, New York: Taylor \& Francis.

31. Guest G. Applied thematic analysis. Thousand Oaks, California: Sage 2012.

32. Schnittker R, Marshall SD, Horberry T, Young K (2019) Decision-centred design in healthcare: The process of identifying a decision support tool for airway management. Applied Ergonomics. 77, pp 70-82

33. Donabedian A. (2003) An introduction to quality assurance in health care. New York, NY: Oxford University Press.

34. McGaghie W, Miller GE, Sajid AW, et al. (1978) Competency-based curriculum development on medical education: an introduction. Am J Public Health;68:11-91.

35. McGaghie et al (2011) Medical Education Featuring Mastery Learning With Deliberate Practice Can Lead to Better Health for Individuals and Populations. Academic Med. 86(11):e8-e9

36. Wayne DB, Butter J, Siddall VJ, et al. (2006) Mastery Learning of Advanced Cardiac Life Support Skills by Internal Medicine Residents Using Simulation Technology and Deliberate Practice. J Gen Intern Med;21(3):251-256.

37. RCoA Simulation Strategy (2019) Royal College of Anaesthetists Simulation Strategy 2018-2023. https://www.rcoa.ac.uk/sites/default/files/documents/2019-07/Simulation-Strategy-FINAL.pdf Accessed 02/02/2020

38. Sauter TC, Hautz WE, Hostlettler S, Brodmann-Maeder M, et al (2016) Interprofessional and interdisciplinary simulation-based training leads to safe sedation procedures in the emergency department. Scandinavian Journal of Trauma, Resuscitation and Emergency Medicine. 24, article no: 97.

39. Marshall SD \& Touzell A (2020) Human Factors and the safety of surgical and anaesthetic care. Anaesthesia, 75(suppl.1), e34-38.

\section{Figures}


1. Recognition of endotracheal tube displacement (i.e. visual, auditory)

2. Call for help (i.e. shout, emergency buzzer)

3. Oxygenate patient

3.1. Grab oxygen mask or Water's circuit

3.2. Place mask on patient

3.3. Attach to oxygen supply

3.4. Conduct basic airway manoeuvres

4. Check breathing
4.1. Check for mist on mask
4.2. Check oxygen saturation
4.3. Check chest movement
4.4. Check Water's circuit bag for movement (if applicable)
4.5. Auscultate chest
4.6. Suction to clear airway (if necessary)

5. Airway trained doctor arrives

5.1. Turn off ventilator alarm (if necessary)

5.2. Brief patient summary (if necessary)

6. Decision made to reintubate or not

6.1. Routine oral endotracheal intubation or reintubation via tracheostomy (if applicable)

7. Identify emergency equipment (i.e. bag, trolley)

7.1. Check availability of supplies

7.1.1. Laryngoscope blades

7.1.2. Light handles

7.1.3. Extra endotracheal tubes

7.1.4. Bougies, guide wires

7.1.5. 1-gels

7.2. Check suitability (i.e. correct size, unopened)

8. Reintubate

8.1. Administer drugs (if necessary)

8.2. Insert endotracheal tube

8.2.1. Laryngoscope blade to visualise larynx

8.2.2. Pass bougie through tracheostomy (if applicable)

9. Check position of endotracheal tube

9.1. Reapply Water's circuit or ventilator

9.2. Check breathing

9.2.1. Check mist on tube

9.2.2. Check oxygen saturation

9.2.3. Check chest movement

9.2.4. Check capnography (if available)

9.3. Return patient to ventilator (if necessary)

9.4. If problem identified, proceed to task 8

10. Secure endotracheal tube

10.1. Place Anchorfast on patient

10.2. Tape tracheostomy tube (if applicable)

\section{Figure 1}

Hierarchical Task Analysis of Unplanned Extubations 


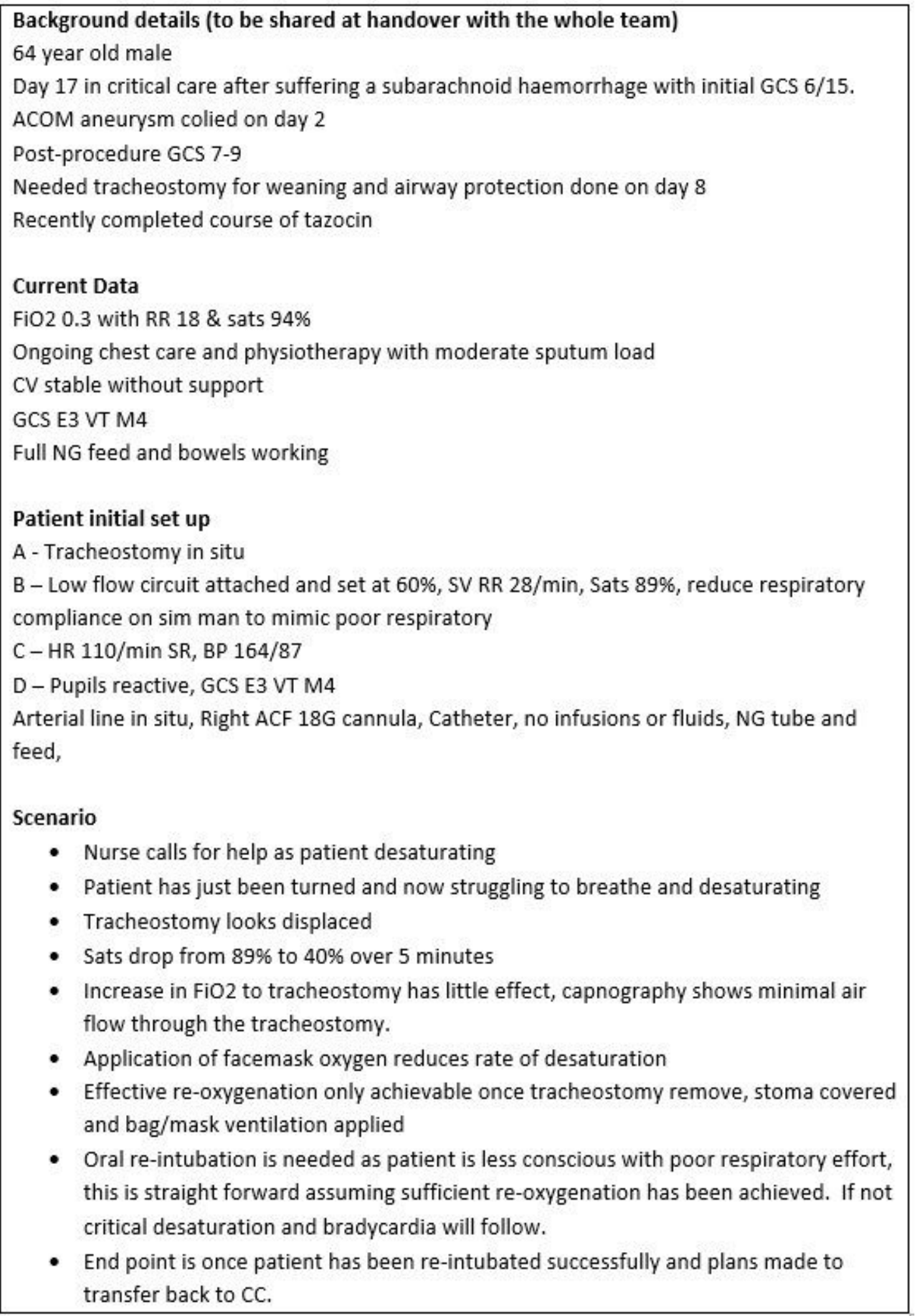

\section{Figure 2}

Example Simulation UE Scenario 


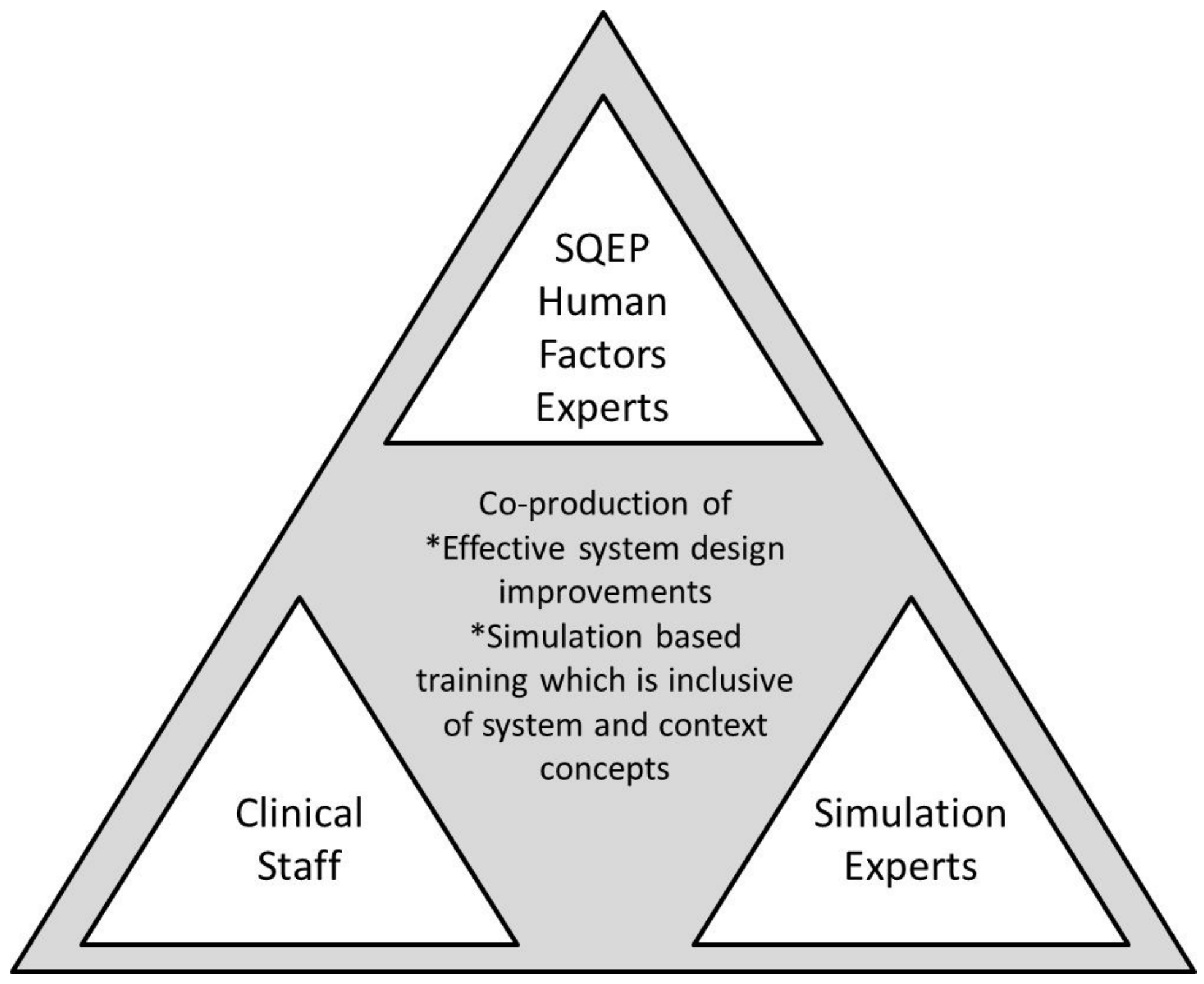

Figure 3

Recommended triad of expertise for investigation and development of safety interventions in acute care 